\title{
The Development of Advanced Composite Material with Metal Adhered by an lonic Bond to the Surface of a Woody Biomass
}

\author{
Manabu NAKAGAWASAI, Shigeaki INADA, \\ Hisanobu KAWASHIMA, Shigeki MATSUOKA, \\ Yusuke UENO and Tatsuya HOSHI \\ Department of Mechanical Systems Engineering, Gunma University, \\ 1-5-1, Tenjincho, Kiryu-shi, Gunma 376-8515, Japan
}

Keywords: Woody Biomass, Plasma Irradiation, Metal Adhesion, Water Absorbency, Silver Ion, Sodium Ion

The purpose of this study is to adhere titanium, sodium and silver to the surface of woody biomass by low temperature plasma irradiation. As the woody biomass, wood powder and coffee grounds were used. First, those powder samples were ozonized by oxygen plasma irradiation. $\mathrm{Next} \mathrm{TiCl}_{4}$ gas, $\mathrm{NaNH}_{2}$ gas or $\mathrm{AgNO}_{3}$ gas plasma was generated in a quartz tube and the sample was irradiated in the tube. The composition of the sample surface was identified using an XPS. In the result, it was confirmed that three kinds of metals bonded to the wood powder. The hydrophilic property, photocatalyst, and sterilization characteristic of the wood powder were examined.

\section{Introduction}

Developments in scientific knowledge have shown that, dioxins (Kim et al., 2001) and heavy metal, etc. are suspected causes soil pollution. As a countermeasure for harmful trihalomethane (Bellar et al., 1974; Gray, 1994) which was produced by a reaction with organic substances in a process of chlorine sterilization treatment of water, the development of a new sterilization method is desired as a substitute for a chlorine.

Traditionally, when woody biomass was utilized, each component of lignin, hemicellulose and cellulose was perfectly separated, and then, each separated component was put to use. The component separation is carried out by extraction using heat, alkali and organic solvent, hydrolysis using acid and the combination (Minowa et al., 1998; Nakamura et al., 2001), however, one of the drawbacks to this method is that the treatment running cost is high due to the complication of the many treatment processes and in addition, results in damage of the components.

In this study, wood was made first of all into wood powder, and as the first plasma exposure process, oxygen plasma was directly irradiated on the powder under conditions in which the components were not sepa-

Received on May 22, 2007; accepted on October 5, 2007. Correspondence concerning this article should be addressed to S. Inada (E-mail address: inada@me.gunma-u.ac.jp).

Presented at International Symposium on Innovative Materials for Processes in Energy Systems, IMPRES, Kyoto, October, 2007. rated. In this process under dry conditions, the lignin component is oxidized by the strong oxidizability of ozone and becomes porous so that the functional group, which is rich in oxygen, becomes easy to be modified on the lignin surface as well as the cellulose. Next, as the second plasma exposure process, when the ozonated wood powder is exposed to a gas plasma containing metal such as sodium, titanium and silver, the metal combines with the wood powder.

Conventionally, a graft polymerization technique was adopted so that wood powder was chemically made to combine inorganic material and plastic, after the lignin component was perfectly removed (Hamada, 2002). In such graft polymerization, a wet process using solvent was predominantly used, there were many treatment processes, the operation was also complicated, and the treatment running cost was also high. On the other hand, the graft polymerization method which introduced various functional groups into the backing polymers such as polyethylene, polypropylene and cellulose as a monomer has been recognized as a dry method using gamma-ray radiation (Furusawa, et al., 2005; Wada et al., 2006). However, at the present, the fact that the small radiation exposure quantity is not dangerous to the human body, has not yet been widely accepted. So, the use of the radiation is apt to be avoided. On the other hand, the low-temperature plasma irradiation used in this study is a glow discharge plasma in the wavelength region of visible light, and there is completely no danger of plasma exposure or negative effects on the human body. In addition, by using strong electronic energy, graft polymerization under dry conditions is possible. 
The purpose of this study is to create new clarification materials at low-cost in order to solve these problems.

At present, reuse of wood-based waste and utilization of woody biomass are being reexamined. Woody biomass is an organic substance which can be cheaply obtained, and especially its utilization as a bulk material is effective, since it decomposes in nature. If the woody biomass possesses the functions of photocatalyst, dehalogenation and sterilization; the application over a wide field becomes possible, and its commercial development can be expected. As the carrier which adsorbs the metal, porous active carbon and zeolite, etc. are generally utilized, when the adsorption efficiency is considered to be a primary priority. However, in this study, wood powder and coffee grounds were used as carriers, and the metal was adsorbed unconventionally on the surface of the carrier by the following methods. First, the powder samples were ozonized by oxygen plasma irradiation. Next, gas plasma using titanium tetrachloride $\mathrm{TiCl}_{4}$, sodium amide $\mathrm{NaNH}_{2}$ or silver nitrates $\mathrm{AgNO}_{3}$ as a precursor was generated in a quartz tube and the sample was irradiated in the tube. The composition of the sample surface was identified using an XPS (X-ray photoelectron spectroscopy). If titanium was captured by the ionic bond on the surface of the woody biomass, it is expected to be utilized in environmental cleanup technology for neutralizing hazardous chemical. If sodium attaches to the woody biomass, it becomes useful as a dehalogenate countermeasure material. If silver attaches to the woody biomass, and the silver ions appear when it is mixed with water, the development of a new sterilization method can be expected as a substitute for chlorine.

\section{Experiment}

\subsection{Experimental apparatus}

The schematic of experimental equipment is shown in Figure 1. The experimental apparatus is composed of a quartz tube that plasma is generated from, the induction coil around the tube, a $360 \mathrm{kHz}$ high frequency power supply, exhaust equipment to create a vacuum, Pirani gauge, cooling water supply equipment and a neutralization tank to capture the poison gas. The reaction tube is made from quartz, and is $300 \mathrm{~mm}$ in its outside diameter, $290 \mathrm{~mm}$ in its internal diameter, and a cylinder with a length of $1000 \mathrm{~mm}$. This equipment is an external induction type low-temperature plasma generator. An oil rotary pump was used for the exhaust in the furnace.

Qualitative and quantitative analyses were performed by using the XPS. Surface observation was performed by using an SEM (scanning electron microscope). The intensity distribution at each wavelength of infrared light was examined using the FT-IR (Fourier transform-infrared spectroscopy) method in order to

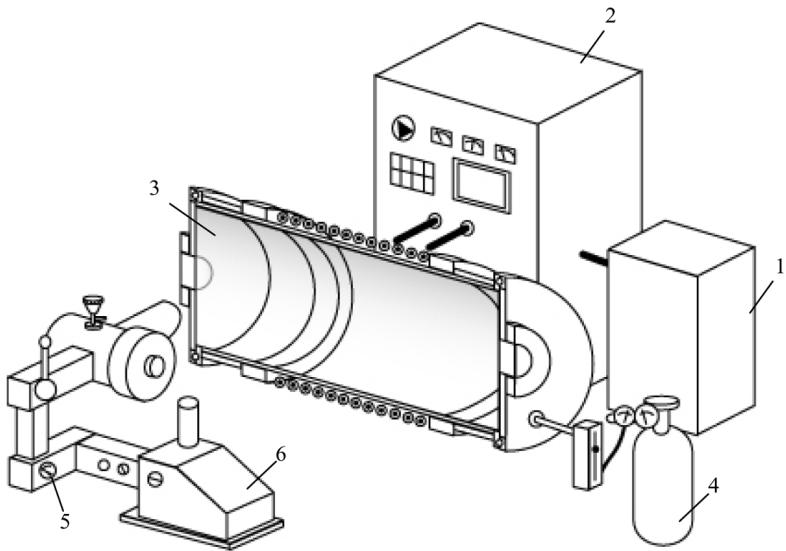

Fig. 1 Experimental apparatus: 1, Water tank and water pump; 2, High frequency power supply; 3, Quartz tube; 4, Oxygen gas cylinder; 5, Pirani vacuum gauge; 6 , Oil rotary vacuum pump

evaluate the functional group modified on the surface of the wood powder.

\subsection{Experimental procedure}

The conifer wood chips were broken down by a grinding mixer, and processed into wood powder of diameter and length of about $0.5-1 \mathrm{~mm}$. Drink residue of ground coffee of a marketed drip type was used for the experiment, and it was sufficiently dried.

In the first irradiation process, oxygen gas of high purity (G2 class; $99.999 \%$ ) was sent to the plasma reaction tube at a rate of $1000 \mathrm{~mL} / \mathrm{min}$. The pressure in the reaction tube was retained by a balance between supply of the oxygen gas and exhaust by the vacuum pump at about 5 Torr. At this time, a plasma glow discharge of a light purple color was uniformly started in the furnace, when a $3 \mathrm{kV}$ high frequency anode voltage was applied. Beforehand, about $5 \mathrm{~g}$ of wood powder thinly and uniformly sprayed on a flat quartz plate was inserted as it was in the furnace.

Judging from a multi-measurement of the XPS, it was proven that a relative content rate of carbon atoms and oxygen atoms, which are main components of the wood powder, changed according to the exposure time of the oxygen plasma to the wood powder. With the exposure time, the relative content rate of carbon atoms decreased, and oxygen atoms reversely increased. However, it was proven that both elements maintained a constant value when the exposure time was set over $120 \mathrm{~min}$, according to the experimental result for the exposure time of 6 stages from 5 to $150 \mathrm{~min}$. In the same way, in the second irradiation process, it was proven that the amount of metallic adsorbed to the wood powder maintained a constant value when the exposure time was set over $30 \mathrm{~min}$. Based on the above measurement results, 120 min was taken as the ozonation processing time, and the metal adsorption processing time for $30 \mathrm{~min}$ was set. 
The titanium tetrachloride gas plasma, the sodium amide gas plasma and the silver nitrate gas plasma were generated so that titanium, sodium and silver may respectively be captured by ionic bond on the surface of the woody biomass. For the powdered materials of silver nitrate and sodium amide, those specified quantity of 3-10 g were laid on a steel plate, and the steel plate itself was inserted in the plasma furnace. The powdered material was melted and gasified through the steel plate heated by a high frequency induction effect in order to generate vaporization gas plasma of silver and sodium, respectively. In the case of the liquid titanium tetrachloride, the liquid was vaporized by a vaporization promoting effect under decompression, after $100 \mathrm{~mL}$ of the liquid was poured in a beaker inserted in the furnace. The above-mentioned loading quantity was chosen as an appropriate quantity of the precursor, because most of the vaporized material is uselessly exhausted, if the quantity loaded in the furnace is too much.

In this experiment, the relative content rate of the composition atoms of the wood powder and adsorbed metal can be measured by the XPS, however, it is impossible to measure the amount of metal itself adsorbed to the wood powder.

Irradiated samples were used for the XPS measurement, after they were washed in distilled water. An Al-monochrome was used as an excitation X-ray source, for the XPS. The measured voltage and electric power was $15 \mathrm{kV}$ and $400 \mathrm{~W}$. Surface vapor deposition to the sample and the use of an ion gun were not conducted.

\section{Results and Discussion}

\subsection{Confirmation of the metal support quantity by the XPS measurement}

The XPS measurement results of the irradiated wood powder sample for each plasma show the relationship between the spectral intensity and the binding energy in Figures 2-4. By irradiating each gas plasma of titanium, sodium and silver as a precursor, each spectral peak of titanium, sodium and silver newly appeared in Figures 2-4, respectively. In the case of the original samples, peaks of silver, sodium, or titanium did not appear except for oxygen and carbon.

In Figure 2, a spectral peak of chlorine did not appear. This is because the chlorine was removed during the water purification after the titanium tetrachloride gas plasma irradiation. The nitric peak could not be confirmed from Figure 3. Similarly, the nitric peak did not appear in Figure 4 for the silver nitrate gas plasma irradiation.

For four kinds of plasma exposure including the unirradiated original, each concentration of oxygen, carbon, titanium, sodium, and silver in the wood powder sample is shown in Table 1.
Table 1 Result of multi measurement (wood chips) [unit:\%]

\begin{tabular}{lcrrrrr}
\hline & Original & \multicolumn{1}{c}{$\mathrm{O}_{2}$} & $\mathrm{TiCl}_{4}$ & $\mathrm{NaNH}_{2}$ & $\mathrm{AgNO}_{3}$ & $* \mathrm{AgNO}_{3}$ \\
\hline $\mathrm{O} 1 \mathrm{~s}$ & 19.13 & 55.57 & 37.81 & 27.35 & 27.88 & 27.28 \\
$\mathrm{C} 1 \mathrm{~s}$ & 80.87 & 44.42 & 58.50 & 68.47 & 70.86 & 72.72 \\
$\mathrm{Ti} 2 \mathrm{p}$ & 0.00 & 0.00 & 3.22 & 0.03 & 0.00 & 0.00 \\
$\mathrm{Na} 1 \mathrm{~s}$ & 0.00 & 0.00 & 0.16 & 4.15 & 0.00 & 0.00 \\
$\mathrm{Ag} 3 \mathrm{~d}$ & 0.00 & 0.00 & 0.00 & 0.00 & 1.27 & 0.75 \\
\hline
\end{tabular}

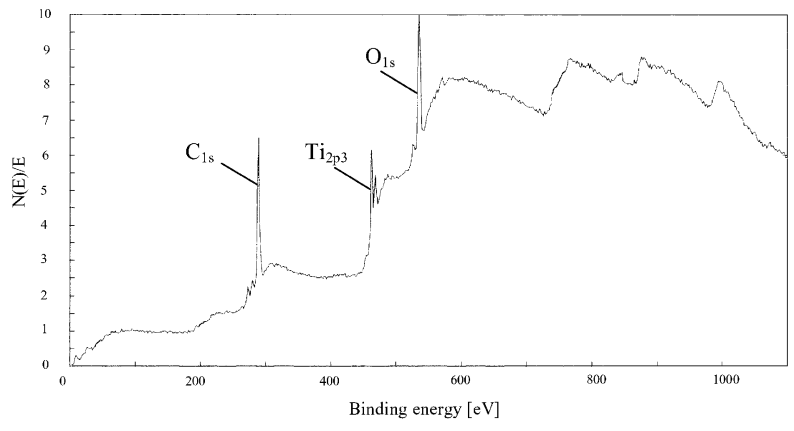

Fig. 2 Wood powder after $\mathrm{TiCl}_{4}$ plasma irradiation

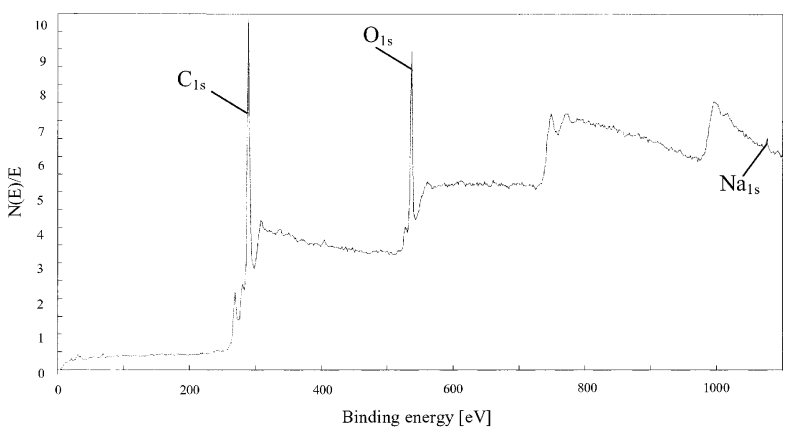

Fig. 3 Wood powder after $\mathrm{NaNH}_{2}$ plasma irradiation

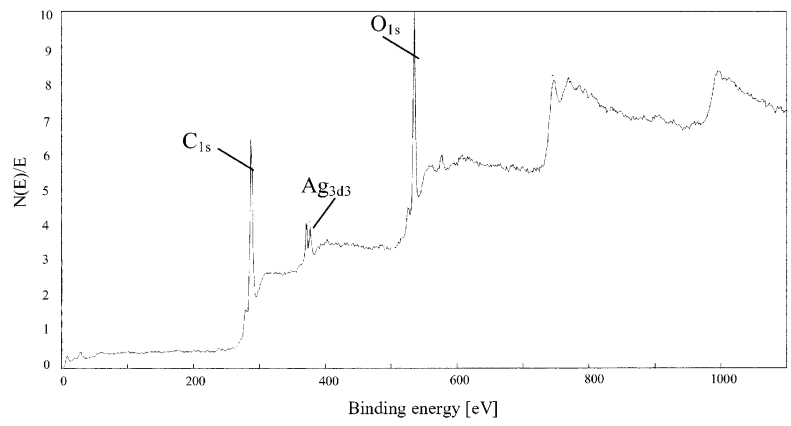

Fig. 4 Wood powder after $\mathrm{AgNO}_{3}$ plasma irradiation

Bonding of the metal to the surface of the cellulose becomes possible by the covalent bond of the cellulose molecule and the metal. However, the lignin component works to block the covalent bond. Then, the lignin must be fixed by the strong oxidizing ability 
Table 2 Result of multi measurement (coffee grounds) [unit:\%]

\begin{tabular}{lrrrrr}
\hline & Original & \multicolumn{1}{c}{$\mathrm{O}_{2}$} & $\mathrm{TiCl}_{4}$ & $\mathrm{NaNH}_{2}$ & $\mathrm{AgNO}_{3}$ \\
\hline $\mathrm{O} 1 \mathrm{~s}$ & 11.13 & 16.85 & 13.91 & 17.22 & 15.18 \\
$\mathrm{C} 1 \mathrm{~s}$ & 88.86 & 83.13 & 85.52 & 82.22 & 84.68 \\
$\mathrm{Ti} 2 \mathrm{p}$ & 0.01 & 0.01 & 0.52 & 0.00 & 0.01 \\
$\mathrm{Na} 1 \mathrm{~s}$ & 0.00 & 0.01 & 0.03 & 0.56 & 0.02 \\
$\mathrm{Ag} 3 \mathrm{~d}$ & 0.00 & 0.00 & 0.02 & 0.00 & 0.10 \\
\hline
\end{tabular}

of ozone. From Table 1, in the case of the oxygen plasma irradiation, an increase of $36 \%$ in oxygen concentration was confirmed in comparison with the original. From this fact, it can be sufficiently assumed that the lignin component in the wood powder was ozonized. As a result, the target metal was adsorbed to the wood powder at $1-4 \%$ content rate.

The composition rate of $* \mathrm{AgNO}_{3}$ in Table 1 shows the case in which ozonation process of the wood powder by the oxygen plasma irradiation was omitted and the wood powder was directly irradiated by gas plasma using $\mathrm{AgNO}_{3}$. Silver inclusion concentration in this case was $0.75 \%$. Because of this, it was proven that the adhesion of the metal to the wood powder was not sufficiently achieved, when the oxygen plasma irradiation process was skipped. This means that the ozonation treatment of the lignin is one of the important processes for adhesion of the metal.

For the coffee residue, the result of carrying out the procedure in the same way as the wood powder is shown in Table 2. From this table, the metallic inclusion rate was only $1 \%$ or less. The reason for this is because the lignin component of the coffee is low in the following. The component of the coffee grounds was cellulose $10 \%$ and lignin $5 \%$ generally, and was under $1 / 5$ of the wood powder.

\subsection{Absorbency evaluation of the sample}

In general, a functional group which has a highhydrophilicity is modified on a sample surface, when the sample is once exposed by oxygen plasma. All samples were put into a beaker filled with water, and the absorbency of the sample was confirmed by observing the sedimentation behavior in the water.

By the following methods, the hydrophilicity of the sample was evaluated. On the level where about $2 / 3$ of a $200 \mathrm{~mL}$ glass container was filled with distilled water, wood powder samples of $3 \mathrm{~g}$ each were gently dropped in. At this time, the time until $90 \%$ of the dropped wood powder reached the base of the container was measured. About $10 \%$ of the wood powder was excluded, because it stagnated on the surface because of an adhesion of dust and a pile of the powder fellow. The hydrophilicity time is shown in Figure 5 for the original non-plasma exposure, the oxygen plasma irradiation sample and the wood powder in which each metal adsorbed. In the case of the original

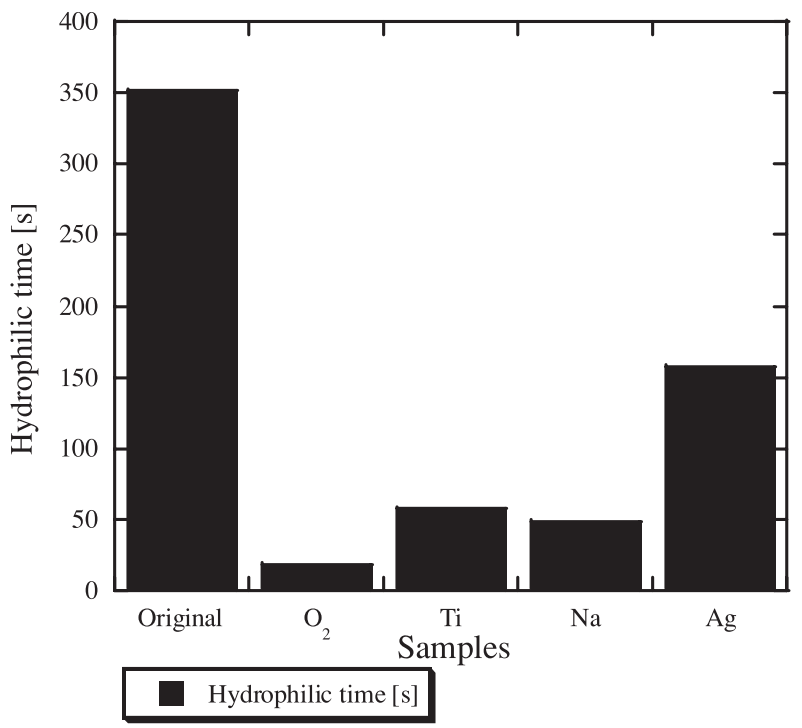

Fig. 5 The evaluation of the hydrophilicity (the time until the wood powder sinks to the bottom of the container)

wood powder, it took $352 \mathrm{~s}$ for the wood powder to reach the container base. On the other hand, it was proven that the hydrophilicity performance was rapidly improved, when this original wood powder was irradiated by oxygen plasma. Then, it was confirmed by the FT-IR measurement that the functional group which had a high hydrophilicity was modified on the sample surface.

In the original wood powder shown in Figure 6(a), there is no remarkable functional group. In the case of the oxygen plasma irradiation shown in Figure $6(\mathbf{b})$, the peak of the $\mathrm{C}-\mathrm{O}$ combination appeared in the vicinity of the wave number of $1030 \mathrm{~cm}^{-1}$ and the peak of the benzene ring in the vicinity of the $1500 \mathrm{~cm}^{-1}$, the peak of $-\mathrm{COOH}$ (carboxyl group) in the vicinity of $2900 \mathrm{~cm}^{-1}$ and the peak of $-\mathrm{OH}$ (hydroxyl group) in the vicinity of $3300 \mathrm{~cm}^{-1}$ appeared respectively.

When the surface of the wood powder is irradiated by low-temperature plasma, the $\mathrm{C}-\mathrm{H}$ coupling of the surface is cut off by a strong electronic energy of plasma. The $\mathrm{H}$ atom is immediately pulled out, because it is light. Then, the oxygen molecule itself forms ozone by combining with an oxygen atom, when the oxygen gas is introduced as a precursor of the plasma. Simultaneously, active species radical such as $\cdot \mathrm{OH}, \cdot \mathrm{H}$ and $\mathrm{O}_{2}^{-}$are formed in large quantities in the plasma reactor. Functional groups which are rich in oxygen such as, $-\mathrm{CO}-,-\mathrm{COOH},-\mathrm{OH}$ are modified on the surface of the wood powder where $\mathrm{H}$ atom was pulled out.

In the second plasma exposure process shown in Figures 6(c)-(e), the combination of the modified functional group and metal was realized. For example, one of the combinations of a carbonyl group of the divalence was replaced with metal, and a hydrogen 


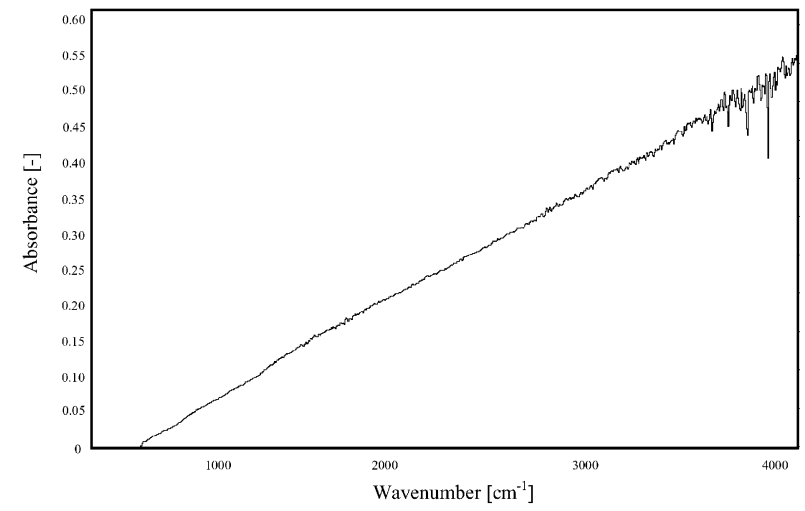

Fig. 6(a) The functional group of the original wood powder

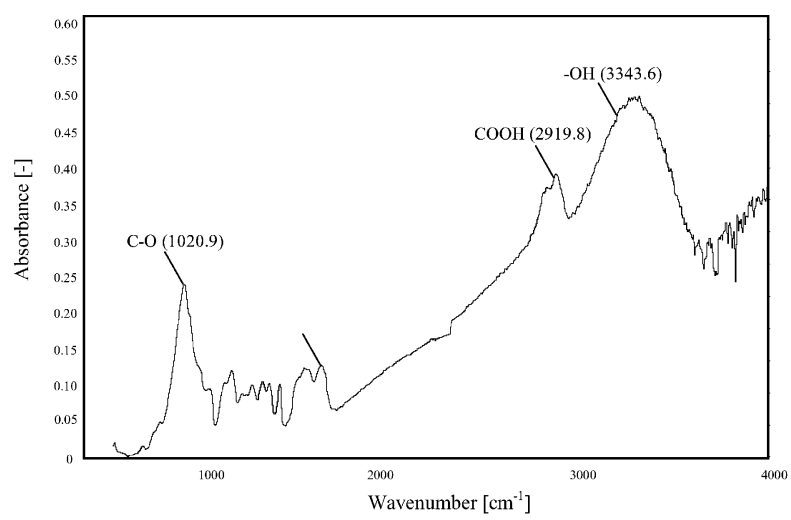

Fig. 6(b) The functional group of the wood powder exposed to the oxygen plasma

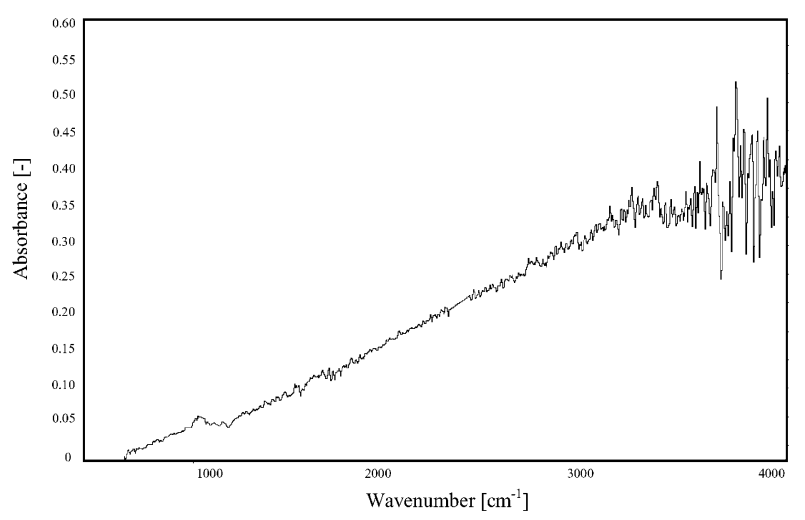

Fig. 6(c)The functional group of the wood powder exposed to the $\mathrm{TiCl}_{4}$ plasma after irradiation of the oxygen plasma

atom of a hydroxyl group and a carboxyl group of the monovalence was also replaced with metal.

In the second plasma exposure process, it means that the metal was substituted at the tip of the functional groups so that the functional group did not appear in the result of the FT-IR method.

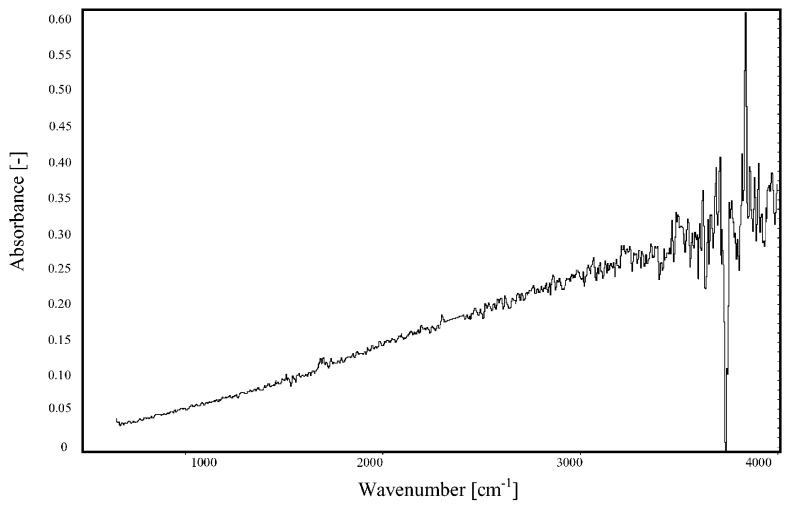

Fig. 6(d) The functional group of the wood powder exposed to the $\mathrm{NaNH}_{2}$ plasma after irradiation of the oxygen plasma

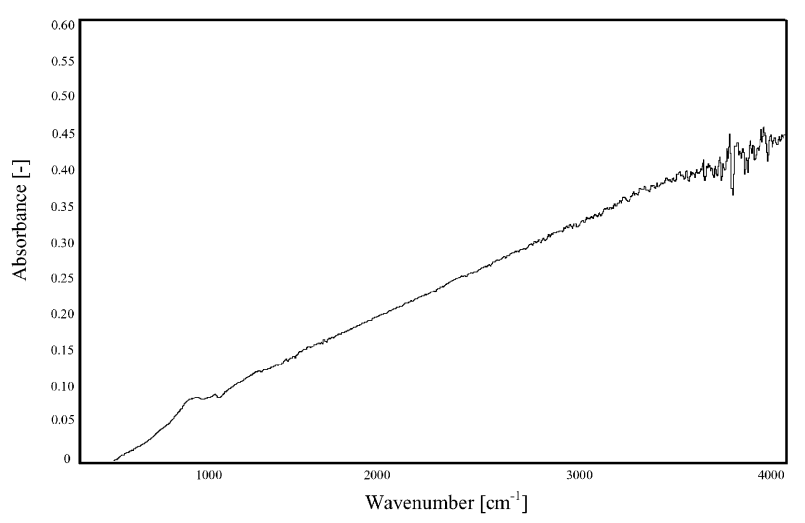

Fig. 6(e)The functional group of the wood powder exposed to the $\mathrm{AgNO}_{3}$ plasma after irradiation of the oxygen plasma

Each of the photographs of Figure 7 shows the sedimentation pattern in the water after $18 \mathrm{~s}$ when the wood powder sample of $3 \mathrm{~g}$ was gently put into the distilled water of $200 \mathrm{~mL}$ in the beaker of $40 \mathrm{~mm} \mathrm{di-}$ ameter and $200 \mathrm{~mm}$ depth. Over $90 \%$ of the sample submerged right after the drop in the cases of oxygen plasma irradiation (b). About $50 \%$ of the sodium containing sample (d) and the titanium containing sample (c) were submerged. About $10 \%$ was merely submerged in the case of the silver containing sample (e). On the other hand, in case of the original sample (a), the most part was still floating on the water surface. The promotion of absorbency could hardly be observed for the coffee sample.

\subsection{Expression of silver ions in the water}

Figure 8 shows the time change of the silver ion concentration in a container filled with the distilled water of $100 \mathrm{~mL}$. Three grams of the wood powder to which the silver was adsorbed were put in the container. The measurement of the silver ion concentration was carried out by installing a silver ion compound sensor 


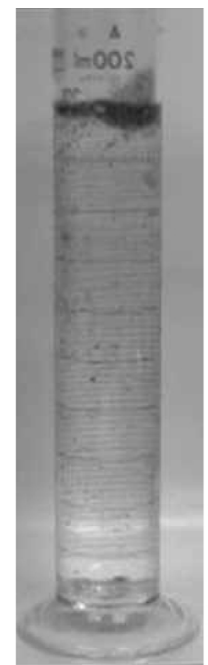

(a)

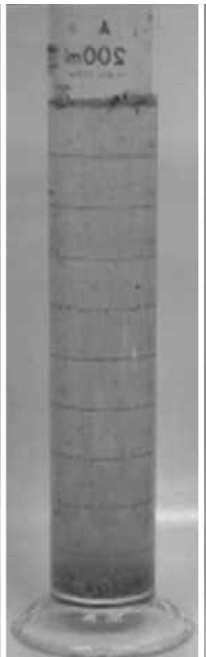

(b)

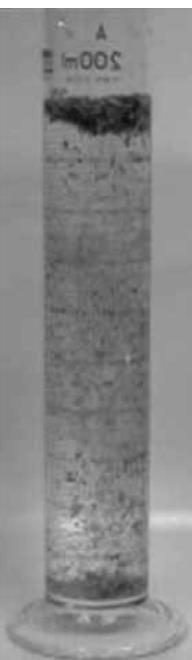

(c)

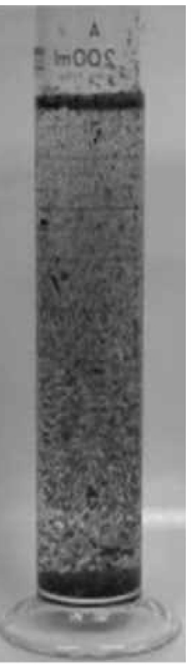

(d)

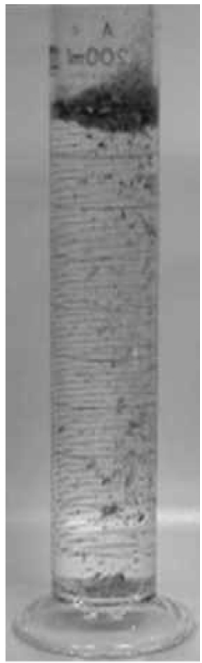

(e)

Fig. 7 Sedimentation pattern of the sample in the water after the dropping: (a) Original wood powder; (b) wood powder irradiated by oxygen plasma; (c) wood powder contained titanium; (d) wood powder contained sodium; (e) wood powder contained silver

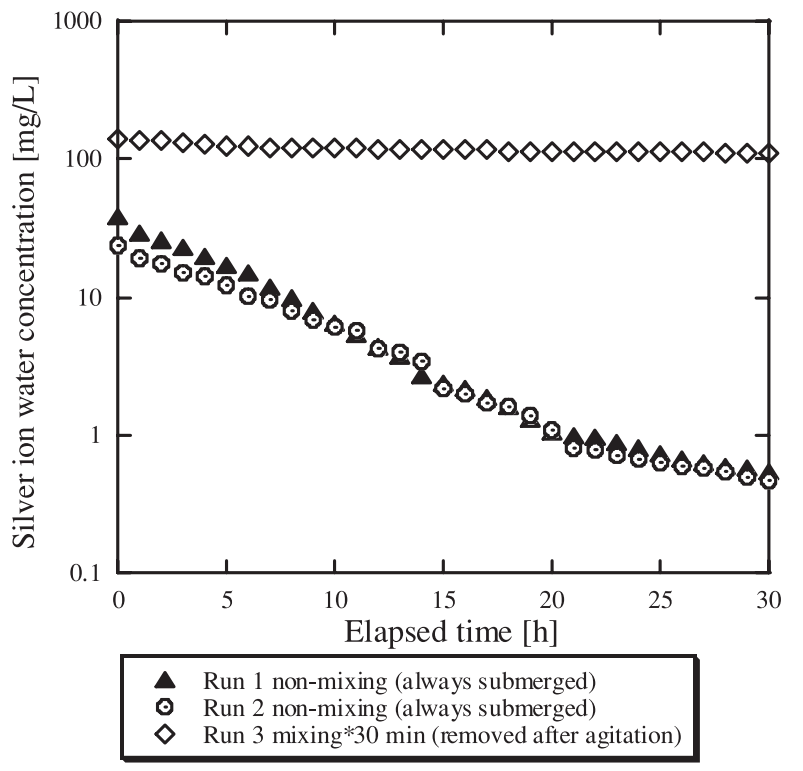

Fig. 8 Time change of the silver ion concentration

(measuring range $0.1-108,000 \mathrm{mg} / \mathrm{L}, \mathrm{Ag}^{+}$) in a portable ion and $\mathrm{pH}$ measuring instrument. When $90 \%$ of the wood powder reached the base of the container, the measurement was started. The first run shown in Figure 8 was a time change of the silver ion concentration measured in this condition. The concentration which showed about $40 \mathrm{mg} / \mathrm{L}$ at the beginning of the measurement decreased with time. The main factor in this decrease seems to be the silver ions reacting with hydroxide ions $\mathrm{OH}^{-}$which appeared in the water and changed to silver oxide after $30 \mathrm{~h}$, the adhesion of a brown solid which seemed to be the silver oxide was confirmed on the container's internal wall. The spent silver carrier on the first run was dried for $24 \mathrm{~h}$ and used again on the second run as shown in Figure 8. The concentration at the beginning of Run 2 decreased in comparison with Run 1, but after $10 \mathrm{~h}$, it was almost identical to the $0.63 \mathrm{mg} / \mathrm{L}$ concentration of Run 1. This means that an active metal species with catalytic property of high reusability was formed in the wood powder. The concentration which corresponds to the $0.63 \mathrm{mg} / \mathrm{L}$ is of a high enough density for sterilization, judging from reports (Kikuchi, 2000; Uchida et al., 2004) that most bacteria are killed at $0.1 \mathrm{mg} / \mathrm{L}$ concentration.

The silver carrier created as well as the sample used in Run 1 was newly put into $100 \mathrm{~mL}$ of purified water and an attenuation characteristic of the silver ion concentration was evaluated. Run 3 shown in Figure 8 is the attenuation characteristic. Before the measurement of Run 3, an agitation of the sample and a commotion of $100 \mathrm{time} / \mathrm{min}$ of the container itself were carried out for $30 \mathrm{~min}$, and the silver carrier itself was removed from the container.

A high silver ion density of $139 \mathrm{mg} / \mathrm{L}$ appeared at the beginning of Run 3 and the silver ion concentration did not attenuate extremely with time, and showed $117 \mathrm{mg} / \mathrm{L}$ after $15 \mathrm{~h}$ and $112 \mathrm{mg} / \mathrm{L}$ after $30 \mathrm{~h}$. This means that the silver ion concentration was almost kept to a constant value, because the reaction changing to silver oxide in the test liquid reached saturation.

Figure 9 shows the change over time of the normalized silver ion water concentration which was made to be $100 \%$ at the measurement start. The quantity of the silver nitrate inserted in the furnace is shown as a parameter. Silver ion concentration in the silver ion 


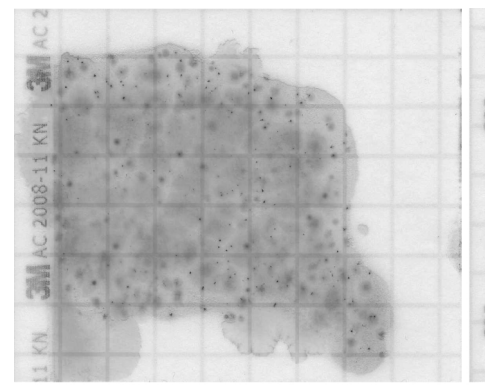

(a)Original

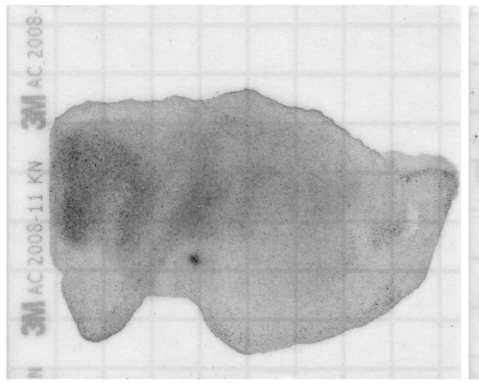

(b)Silver ion water

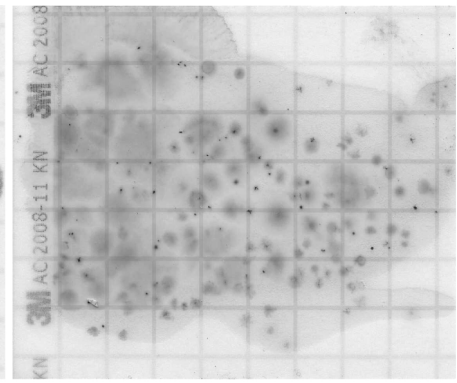

(c)Acid water

Fig. 10 The bactericidal effect of silver ion water and acidic water

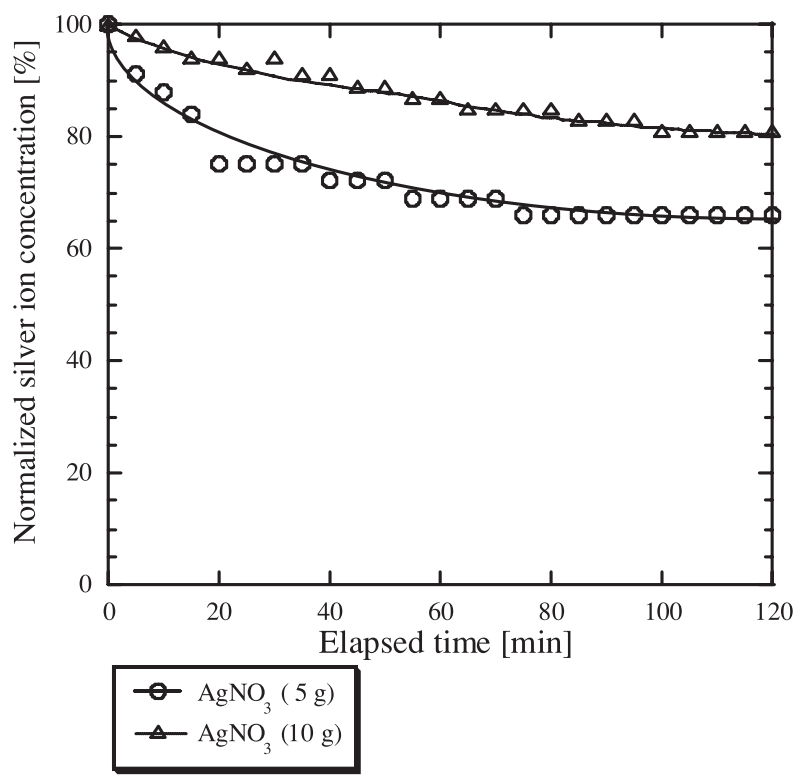

Fig. 9 Time change of the normalized silver ion concentration

water attenuates with time. If the quantity of silver nitrate is abundant, the amount of silver evaporation is also abundant, and as a result, the silver ion concentration is also high. In case of silver nitrate $10 \mathrm{~g}, 80 \%$ of the first concentration was retained even after $2 \mathrm{~h}$, and $75 \%$ was kept even after $24 \mathrm{~h}$ passed. It is important that the vaporization material is effectively adsorbed to the carrier without uselessly exhausting the vaporization material through the vacuum pump.

Silver ions are harmless to the human body, and it is unique in show a strong sterilizing power against most bacteria (legionella genus fungus, salmonella, staphylococcus aureus, pseudomonas aeruginosa, escherichia coli O-157H7, helicobacter Pylori fungus). As a demonstration experiment of bactericidal effect of the silver carrier, it was evaluated using general viable fungus which adhered to black sesame made in Myanmar. First, all adhesive bacteria were discharged in purified water. Then the black sesame was put in the water and was sufficiently stirred. Next, $1 \mathrm{~mL}$ of the water was inoculated on a petrifilm medium, and it was cultivated in an incubator at $35 \AA$ é and carbon dioxide concentration of $5 \%$ for $48 \mathrm{~h}$. By counting the colonies of the fungus, which forms red spots after 48 $\mathrm{h}$, the bactericidal effect was evaluated. Figure 10(a) is a culture of the general viable fungus collected from the black sesame. 157 colonies were confirmed. On the other hand, Figure 10(b) is a result after the general viable fungus was put into silver ion water of 100 ppm for $5 \mathrm{~min}$. Colonies were not observed. It was proven that the fungus completely died in a short time. Figure 10(c) is the result after the general viable fungus was exposed to acidic water adjusted to $\mathrm{pH}=3.46$ for $5 \mathrm{~min}$. Since the silver ion water used was $\mathrm{pH}=$ 3.46 , the effect of the acidity on sterilization was examined. The result was that 111 colonies were detected.

In the silver ion water production method developed in this study, the high density silver ion water was obtained using low electric power of about $300 \mathrm{~W}$. One of its major features is to be able to transport only the necessary quantity to the necessary place since persistency as silver ion water was excellent. The application of silver ion water can be expected at water purifying plants, hospitals, spas, swimming pools, park fountains, cooling towers, and factories for domestic wastewater purification regardless of the size of the installation site. The bactericidal effect of the silver ion originates from the generation of hydrogen radicals and hydroxyl radicals with strong oxidizability.

\subsection{Application field of wood powder which was} combined with sodium

When the wood powder which contains sodium is scattered in soil contaminated by heavy metals and dioxins, etc., the dechlorination of organic compounds including chlorine and PCB can be attempted by the strong reductive reaction in the natural environment. It can also be expected that recalcitrant chemical compounds such as dioxins are decomposed and heavy metals are fixed by use of geochemistry processes such as the crystallization function as a component inside of the crystals.

The wood powder to which sodium was adsorbed was put into purified water $0.1 \mathrm{~g}$ by $0.1 \mathrm{~g}$, and at the 
Table 3 Evaluation of deodorizing function

\begin{tabular}{lcc}
\hline & Before & After \\
\hline Original & 95 & 95 \\
$\mathrm{TiO}_{2}$ carrier & 97 & 33 \\
\hline
\end{tabular}

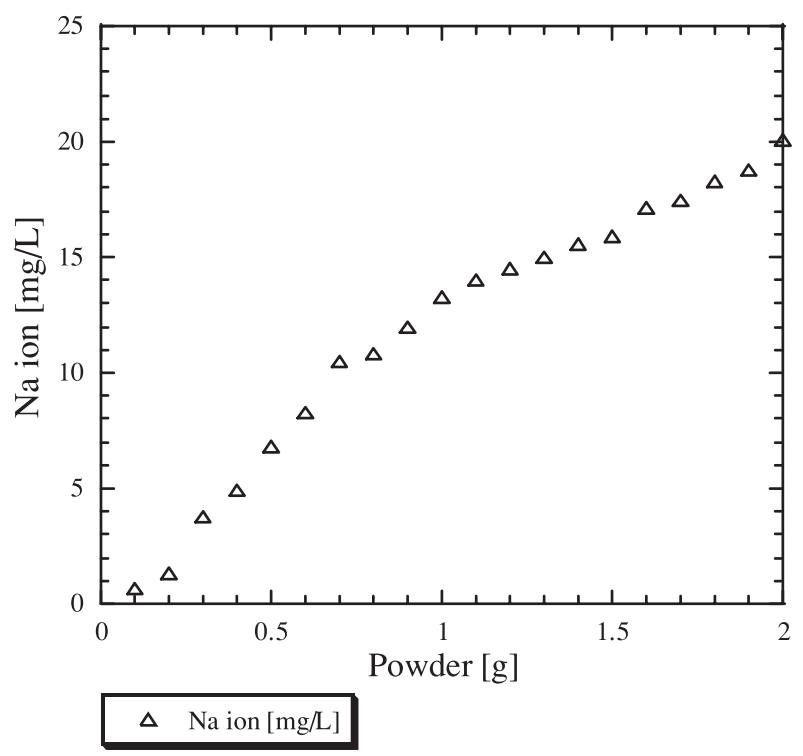

Fig. 11 The expression level of sodium ion in the water

same time the expression level of sodium ion was measured using a sodium ion electrode. The change of the sodium ion concentration against the added amount of sodium carrier is shown in Figure 11. Along with the added amount of the sodium carrier, the concentration of sodium ions rose. A concentration of about $20 \mathrm{mg} / \mathrm{L}$ was observed, when the sodium carrier was increased to $2.0 \mathrm{~g}$ in total.

\subsection{Deodorizing effect of wood powder combined with metal}

The degree of adsorption of odors was measured using a smell monitor (OMX-SR) in order to examine the photocatalyst function of the wood powder to which titanium dioxide was adsorbed. As a measuring method, first, $3 \mathrm{~g}$ of original wood powder and titanium dioxide carrier were inserted in the closed container filled with flue smoke of an incense stick, and the smell was allowed to be adsorbed in the wood powder and the carrier over $60 \mathrm{~min}$. Next, the sampling mouth of the smell monitor was inserted in each type of wood powder and the smell was measured, after the wood powder was taken out from the closed container. The odor intensity at this time is shown in Table $\mathbf{3}$ as before. The odor intensity is indicated by a dimensionless number, and it means that the odor is weak, if the number is small, and the odor remains and is strong, if it is big.
The wood powder was taken out from the closed container, and then, each type of wood powder was exposed to ultraviolet rays of $253.7 \mathrm{~nm}$ wavelength for 3 h. "After" in Table 3 is the odor intensity measured at this time. The output of ultraviolet rays was $4.9 \mathrm{~W}$, and the radiant intensity was $51 \mu \mathrm{W} / \mathrm{cm}^{2}$. In the case of the original wood powder, the odor did not decrease without showing the photocatalyst, even when irradiated with ultraviolet rays. Meanwhile, in the case of the titanium dioxide carrier the odor decreased to about $1 / 3$ of the sample unirradiation by ultraviolet ray. That is to say, most of the odor was decomposed.

The application of wood powder which contains the titania can be expected as an adsorption filter material for tobacco smoke and an aerial purification filter material in hospitals.

\subsection{Surface observation of the wood powder}

Figure 12 shows the observation results by the SEM of each sample's surface. In comparison with the unirradiated original, smoothness had been lost on the surface exposed to the plasmas. In the case of the oxygen plasma irradiation a long stripes pattern appeared, while the whole surface of the metal carrier was seen to be porous. In the case of the sodium carrier, the porous pattern remarkably appeared. It was observed that the titanium carrier was considerably porous, when it was observed at more large magnification as shown in Figure 12(e). From this observation result by the SEM, a specific surface and average pore diameter of the porous surface, etc. could not be quantitatively measured. It is important to note that the carrier is quite porous and able to absorb much metal on the surface so that metal ions or a photocatalyst may appear.

\section{Conclusions}

1. As a result of plasma treatment, titanium, sodium and silver were captured by ionic bond on the surface of the wood powder.

2. As a result of plasma treatment, titanium, sodium and silver bonded to the coffee by ionic bond at only a slight proportion.

3. When the plasma was irradiated on the wood powder sample, the hydrophilicity was improved further than in the case of the unirradiated sample.

4. The absorbency of the sample changed according to the kind of metal adsorbed.

5. The expression of silver ions was confirmed when the wood powder to which the silver was adsorbed was put into water.

6. The bactericidal effect of silver ion water was verified.

7. The expression of sodium ion was confirmed, when the wood powder to which sodium was adsorbed was put into water.

8. The deodorizing effect of wood powder which included titanium was confirmed. 


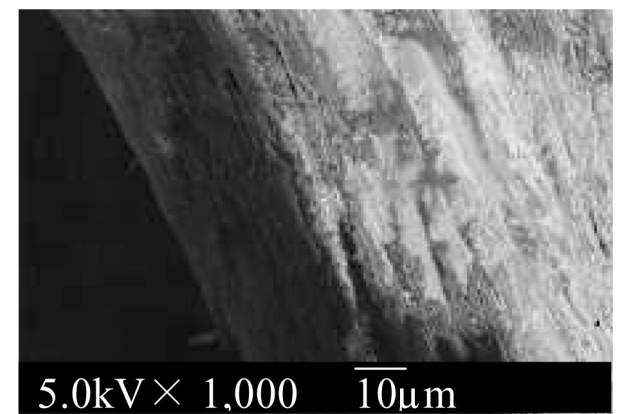

(a) Original wood powder (1000 magnifications)

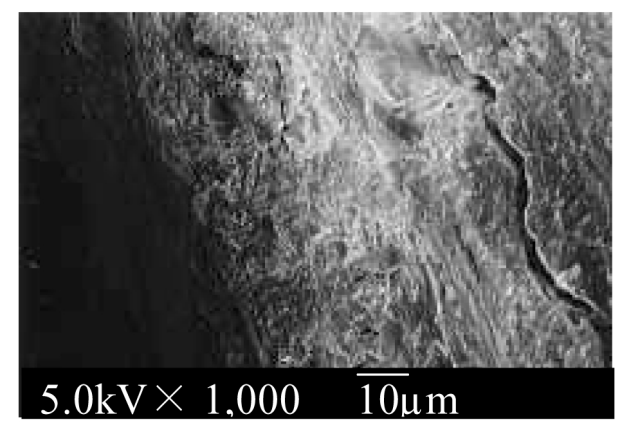

(c) $\mathrm{TiO}_{2}$ carrier (1000 magnifications)

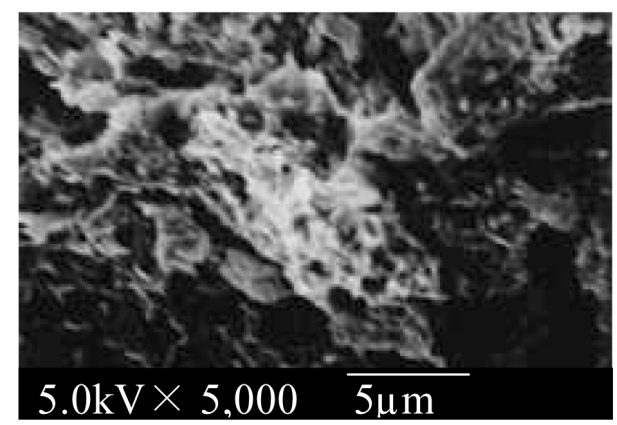

(e) $\mathrm{TiO}_{2}$ carrier (5000 magnifications)

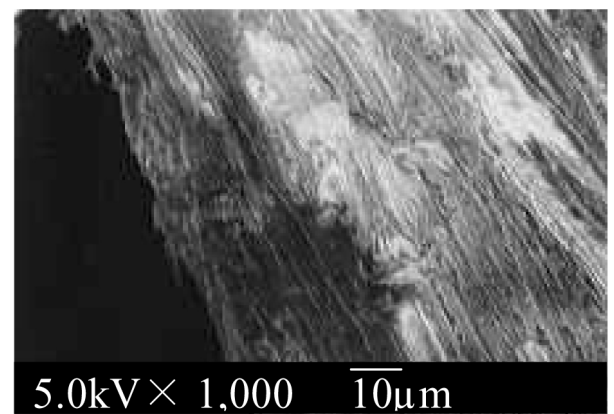

(b) Wood powder exposed to the oxygen plasma (1000 magnifications)

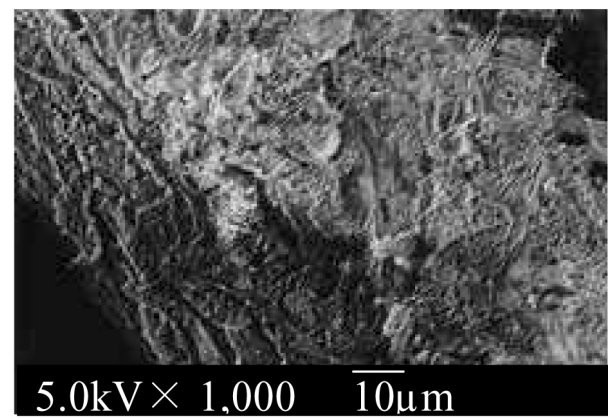

(d) Na carrier (1000 magnifications)

Fig. 12 Surface observation of wood powder

\section{Literature Cited}

Bellar, T. A., J. J. Lichtenberg and R. C. Kroner; "The Occurrence of Organohalides in Chlorinated Drinking Waters," J. American Water Works Association, 66, 703-706 (1974)

Furusawa, K., T. Dobashi, S. Morishita, M. Oyama, T. Hashimoto, N. Shinyashiki, S. Yagihara and N. Nagasawa; "Structural and Kinetic Modification of Aqueous Hydroxy Propyl Methyl Cellulose (HPMC) Induced by Electron Beam Irradiation," Phys. A, 353, 9-20 (2005)

Gray, N. F.; Drinking Water Quality, p. 315, John Wiley \& Sons, Chichester, U.K. (1994)

Hamada, K.; "Practical Use of Wood Materials by Graft Polymerization (Part 1) Graft Polymerization of Methyl Methacrylate onto Wood Using Tetravalent Cerium as Initiator," The Research Report of Kochi Prefecture Industrial Technology Center, 33, 15-19 (2002)

Kikuchi, Y.; "Present State and Problem of Antibacterial Metallic
Material," Materia Japan, 39, 146-150 (2000)

Kim, H. K., H. Kazui, T. Matsumura, K. Ohno, T. Kamei and Y. Magara; "Dioxins in Drinking Water Treatment Process," Organohalogen Compd., 51, 135-138 (2001)

Minowa, T., Z. Fang, T. Ogi and G. Varhegyi; "Decomposition of Cellulose and Glucose in Hot-Compressed Water under Catalyst-Free Conditions," J. Chem. Eng. Japan, 31, 131-134 (1998)

Nakamura, Y., T. Sawada, K. Kuno and Y. Nakamoto; "Resinification of Woody Lignin and Its Characteristics on Safety and Biodegradation," J. Chem. Eng. Japan, 34, 1309-1312 (2001)

Uchida, M., T. Yamamoto, H. Furuhashi, S. Nakata and Z. Nakagawa; "Antibacterial Activity of Silver Ions at a Minimum Inhibitory Concentration," Bokin Bobai, 32, 115-121 (2004)

Wada, Y., H. Mitomo, K. Kasuya, N. Nagasawa, N. Seko, A. Katakai and M. Tamada; "Control of Biodegradability of Poly(3-Hydroxybutyric Acid) Film with Grafting Acrylic Acid and Thermal Remolding," J. Appl. Polym. Sci., 101, 3856-3861 (2006) 